\title{
Nanomechanical Properties and Phase Transitions in a Double-Walled $(5,5) @(10,10)$ Carbon Nanotube: ab initio Calculations
}

\author{
A.M. Popov ${ }^{a *}$, Yu.E. $\operatorname{Lozovik}^{a}$, A.S. Sobennikov ${ }^{b}$, A.A. Knizhnik ${ }^{c}$ \\ ${ }^{a}$ Institute of Spectroscopy, Troitsk, Moscow region, 142190, Russia \\ ${ }^{b}$ Moscow Institute of Physics and Technology, \\ Dolgoprudnyi, Moscow region, 141701, Russia \\ ${ }^{c}$ Russian Research Centre Kurchatov Institute, Moscow, 123182 Russia
}

\begin{abstract}
The structure and elastic properties of $(5,5)$ and $(10,10)$ nanotubes, as well as barriers for relative rotation of the walls and their relative sliding along the axis in a double-walled $(5,5) @(10,10)$ carbon nanotube, are calculated using the density functional method. The results of these calculations are the basis for estimating the following physical quantities: shear strengths and diffusion coefficients for relative sliding along the axis and rotation of the walls, as well as frequencies of relative rotational and translational oscillations of the walls. The commensurability-incommensurability phase transition is analyzed. The length of the incommensurability defect is estimated on the basis of $a b$ initio calculations. It is proposed that $(5,5) @(10,10)$ double-walled carbon nanotube be used as a slider bearing. The possibility of experimental verification of the results is discussed.
\end{abstract}

\section{INTRODUCTION}

In connection with considerable advances made in recent years in the development of nanomechanics, the search for nanoobjects that can be used as mobile elements in nanoelectromechanical systems (NEMSs) has become an urgent problem. The possibility of relative motion of the walls [1, 2] in multi-walled carbon nanotubes makes it promising to use nanotube walls as mobile elements of NEMSs. At present, nanomotors in which the walls of a multi-walled nanotube play the role of the shaft and the bush [3, 4] and memory cells operating on relative motion of the walls along the nanotube axis [5] have been achieved experimentally. A number of NEMSs have been proposed, which operate on the relative motion of carbon nanotube walls, including a gigahertz oscillator [6], Brownian nanomotor 7], and a nut-bolt pair [8, 9, 10]. Nanoelectromechanical systems, such as a varying nanoresistor [9, 10], a stress nanosensor [11] and an electromechanical nanothermometer [12, 13], have also been proposed. The operating principle of these instruments is based on the dependence of the conductivity and the energy of interaction of the walls of a double-walled carbon

\footnotetext{
${ }^{*}$ E-mail: am-popov@isan.troitsk.ru
} 
nanotube (DWNT) on the relative position of the walls on subnanometer scales. Block diagrams and operating principles of such NEMSs were considered in [14]. Thus, analysis of the relative motion and interaction of carbon nanotube walls is very important for developing and designing NEMSs.

The structure of a nanotube wall is controlled by a pair of integers, chirality indices $(n, m)$ corresponding to the coordinates of the carbon lattice vector $\mathbf{c}=n \mathbf{a}_{1}+m \mathbf{a}_{2}$, where $\mathbf{a}_{1}$ and $\mathbf{a}_{2}$ are the unit vectors of the graphite plane. The segment corresponding to this vector becomes the wall circumference when a fragment of the graphite plane is curled to form the nanotube wall [15, 16].

Neither the interaction between carbon nanotube walls nor the interaction between graphite layers have been investigated in details. The results of measurements and theoretical calculations of the energy of interaction between graphite layers differ by two orders of magnitude (see [17] and references therein). The shear strengths along the nanotube axis were measured only in a few experiments [1, 2]. As regards theoretical studies, the barriers for the relative motion of the walls were calculated for a large number of DWNTs using semiempirical potentials [8, 18, 19, 20, 21], as well as ab initio calculations for certain DWNTs [22, 23, 24, 25, 26]. Barriers for the relative motion of the walls of $(5,5) @(10,10)$ DWNT were determined using all methods employed for such calculations. The choice of $(5,5) @(10,10)$ DWNTs was dictated by the following considerations: (i) a small number of atoms in the unit cell of a nanotube, which renders it suitable for ab initio calculations; (ii) a large height of the barrier for the relative rotation of the walls can be observed only for DWNTs with compatible rotational symmetries of the walls [19, 21], including $(5,5) @(10,10)$ DWNT.

Certain semiempirical methods [8, 21], as well as ab initio computational methods [25, 26] used for determining the barriers for the relative motion of the walls of $(5,5) @(10,10)$ DWNT, give values of structural parameters and elastic properties of graphite that are in good agreement with experiment. Nevertheless, the available data for these barriers in Table 1 show that the results of calculations based on different methods differ by several orders of magnitude. Thus, further development of theoretical methods for studying the interaction of layers in carbon nanotubes requires that the barriers for the relative motion of the walls be experimentally determined. Comparison of experimental and calculated heights of these barriers can be used as a criterion for the correctness of the results of calculations.

Here, we will demonstrate that the results of calculating the elastic properties and structure of graphite using the density functional method in the local density approximation with the basis consisting of a set of plane waves agree well with experiment. This method is used for calculating 
the elastic properties, the structure, the energy of interaction, and the height of barriers for the relative motion of the walls in $(5,5) @(10,10)$ DWNT. The results of these $a b$ initio calculations are used to estimate the following physical quantities for $(5,5) @(10,10)$ DWNT: shear strengths and diffusion coefficients for the relative sliding along the axis and relative rotation of DWNT walls, the frequencies of relative rotational and translational oscillations of the walls, and the energy of formation and length of the incommensurability defect. We consider the possible experimental study of these quantities.

It should also be noted that the DWNTs under theoretical investigation in this research were obtained using various synthesis methods: in the standard arc for obtaining carbon nanotubes [27], in the same arc in the presence of hydrogen and a catalyst [28], during catalytic decomposition of hydrocarbons [29], and from single-walled nanotubes with a fullerene chain inside and subjected to heating [30] or bombardment with electrons [31].

\section{COMPUTATIONAL TECHNIQUE}

All calculations based on the density functional method in the local density approximation [32] with the basis consisting of a set of plane waves were performed using VASP (Vienna Ab Initio Simulation Package) [33]. The interaction of valence electrons with atomic cores was described using ultrasoft pseudopotentials [34]. The maximal kinetic energy of plane waves was $358 \mathrm{eV}$. Our analysis shows that the error in calculating the energy of the system is less than $0.001 \mathrm{meV} /$ atom for the chosen cutoff energy for the plane waves. In addition, this error estimate should be insensitive to the structure of the system since the basis of plane waves does not contain an error associated with superposition of the basis function in contrast to the basis of localized orbitals.

Summation over the Brillouin zone was performed over special points of the Monkhorst-Pack type [35]. The structure and elastic properties of graphite were calculated using a set of 172 irreducible $\mathrm{K}$ points in the Brillouin zone. The barriers for the relative sliding and rotation of the walls of $(5,5) @(10,10)$ DWNT were analyzed using a set of nine irreducible K points along the axis of nanotubes.

The structure and elastic properties of graphite were calculated as follows. The lengths of vectors $a$ and $c$ of the fundamental translations of the graphite lattice in the plane of the layers and in the direction perpendicular to this plane varied in the limits $2.39 \AA \leq a \leq 2.49 \AA$ and $6.57 \AA \leq c \leq 7.90 \AA$, respectively. The calculated dependence of total energy $U_{g}$ of the system on the length of the fundamental translation vectors of the graphite lattice was approximated by 
a third-degree polynomial,

Table 1 Barriers $\Delta U_{z}$ and $\Delta U_{\phi}$ for relative sliding of the walls along the axis and relative rotation of the walls about the $(5,5) @(10,10)$ DWNT axis per atom of the outer wall and ratio $\gamma_{b}=\Delta U_{\phi} / \Delta U_{z}$ of these barriers

\begin{tabular}{|l|c|c|c|}
\hline Reference & $\Delta U_{z}, \mathrm{meV} /$ atom & $\Delta U_{\phi}, \mathrm{meV} /$ atom & $\gamma_{b}$ \\
\hline$[8]^{a}$ & 0.008 & 0.025 & 3.13 \\
{$[21]^{b}$} & 0.00745 & 0.0144 & 2.90 \\
{$[20]^{c}$} & 7.5 & 8.7 & 1.16 \\
{$[24]^{d}$} & 0.128 & 0.438 & 3.47 \\
{$[23]^{d}$} & - & 1.2 & - \\
{$[22]^{e}$} & 0.35 & 0.78 & 2.26 \\
{$[25]^{f}$} & 0.125 & 0.259 & 2.08 \\
{$[26]^{g}$} & 0.138 & 0.407 & 2.85 \\
Our data $^{e}$ & $0.005 \pm 0.001$ & $0.205 \pm 0.002$ & 41 \\
\hline
\end{tabular}

Note: ${ }^{a}$ Lennard-Jones potential, optimized structure of the walls; ${ }^{b}$ Lennard-Jones potential, nonoptimized structure of the walls; ${ }^{c}$ Crespi-Kolmogorov potential, optimized structure of the walls; ${ }^{d}$ tight binding method; ${ }^{e}$ density functional method, local density approximation, basis set is formed by plane waves; ${ }^{f}$ density functional method, local density approximation, basis set is formed by pseudowave $p d p p$ functions; ${ }^{g}$ density functional method, local density approximation, basis set is formed by pseudowave $d d d d$ functions.

Table 2 Coefficients of approximation of the dependence of the total energy of graphite on the lengths of fundamental translation vectors of the lattice by third-degree polynomial (11)

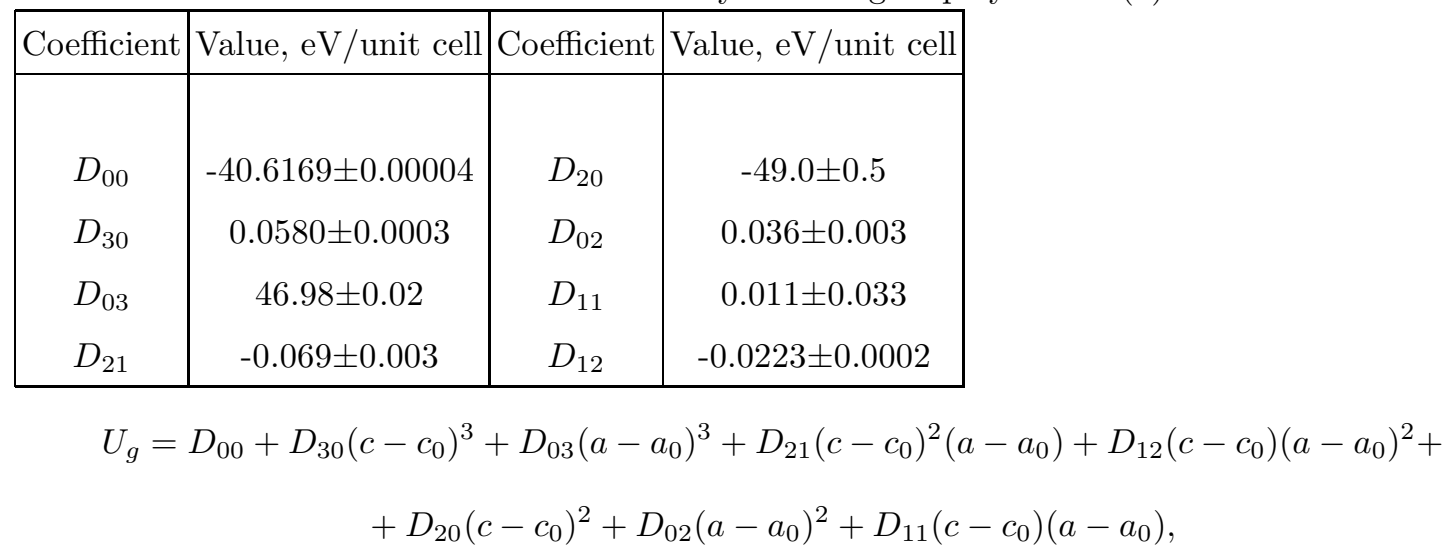

where $a_{0}$ and $c_{0}$ are the equilibrium lengths of the fundamental translation vectors of the graphite lattice. Table 2 gives the values of the coefficients obtained as a result of approximation, while Table 3 gives the values of equilibrium lengths of the fundamental translation vectors of the graphite cell. The elastic constants of graphite were obtained using expression (1) for the total energy of the system, 


$$
C_{11}+C_{12}=\frac{a_{0}^{2}}{2 V_{0}} \frac{\partial^{2} U_{g}}{\partial a^{2}}, \quad C_{33}=\frac{c_{0}^{2}}{V_{0}} \frac{\partial^{2} U_{g}}{\partial c^{2}},
$$

where $V_{0}$ is the equilibrium volume of the unit cell of graphite. Table 3 contains the elastic constants of graphite. In the same table, our results of calculating the structural and elastic properties of graphite with the density functional method in the local density approximation are compared with the results obtained in [36] and the experimental data described in [36]. This comparison shows that the accuracy of our calculations corresponds to the typical accuracy of calculations by the density functional method.

Thus, our calculation method gives values of the lattice parameters and elastic properties of graphite close to experimental ones. We believe that this method can also be used for studying the interaction and relative motion of carbon nanotube walls.

Table 3 Equilibrium lengths of the fundamental translation vectors of the lattice and elastic constants of graphite

\begin{tabular}{|c|c|c|c|}
\hline & Our result & {$[36]$} & Experiment (300K) \\
\hline$a_{0}, \AA$ & $2.44140 \pm 0.00001$ & 2.440 & $2.4608 \pm 0.0016$ \\
$c_{0}, \AA$ & $6.586 \pm 0.002$ & - & - \\
$c_{0} / a_{0}$ & 2.698 & 2.74 & $2.725 \pm 0.001$ \\
$C_{11}+C_{12}, \mathrm{GPa}$ & 1319 & 1283 & $1240 \pm 40$ \\
$C_{33}, \mathrm{GPa}$ & 23.7 & 29 & $36.5 \pm 1$ \\
\hline
\end{tabular}

Table 4 Structural parameters and elastic properties of the $(5,5)$ and $(10,10)$ walls: $R$ is the radius, $t_{s}$ is the equilibrium length of a unit cell, and $E_{s}$ is the Young modulus

\begin{tabular}{|l|c|c|c|c|}
\hline Wall & \multicolumn{2}{|c|}{$(5,5)$} & \multicolumn{2}{|c|}{$(10,10)$} \\
\hline & our result & available data & our result & available data \\
\hline$R, \AA$ & 3.40 & $3.408[26]$ & 6.77 & $6.748[26]$ \\
$t_{s}, \AA$ & 2.439 & $2.4434[26]$ & 2.443 & $2.4420[26]$ \\
$E_{s}, \mathrm{TPa}$ & 1.24 & $0.95[38], 0.96[\underline{39}]$, & 1.31 & $0.92[\underline{38}], 0.98[40]$, \\
& & $1.03[26], 1.06[37]$ & & $1.10[26], 1.24[\underline{41]}$ \\
\hline
\end{tabular}

\section{RESULTS AND DISCUSSION}

First, we calculated the equilibrium structure of isolated $(5,5)$ and $(10,10)$ walls. The structure of each wall was optimized for various lengths $t$ of the unit cell of the wall. The dependence of the total energy $U_{t}$ of the unit cell on its length was interpolated by Hooke law,

$$
U_{t}=U_{s}+\frac{\mu\left(t-t_{s}\right)^{2}}{2}
$$


where $U_{s}$ and $t_{s}$ are the total energy and length of the unit cell, which correspond to the equilibrium structure, and $\mu$ is the elastic coefficient of a wall of length $t_{s}$. The values of $U_{s}, t_{s}$, and $\mu$ were calculated using the least squares method. The Young modulus of the wall was determined in most publications using the expression

$$
E_{s}=\frac{\mu t_{s}}{\pi R w}
$$

where $R$ is the radius of the wall corresponding to the equilibrium structure and $w$ is the effective thickness of the wall: $3.4 \AA$ [26, 37, 38, 39, 40, 41]. Table 4 gives the calculated values of the structural parameters and the Young modulus of the $(5,5)$ and $(10,10)$ walls, as well as the corresponding data from the literature. It should be noted that the calculated lengths of the bonds are in conformity with the results of other calculations based on the density functional method 26], while the calculated values of the Young modulus match those calculated using the density functional [26, 38, 39], Hartree-Fock 37], and tight binding methods [40, 41].

The difference in the unit cell lengths of the $(5,5)$ and $(10,10)$ walls, $\Delta t=t_{2}-t_{1}=0.004 \AA$, is three orders of magnitude smaller than the unit cell lengths. However, a wall with a smaller unit cell length can be extended, while that with a larger unit cell length can contract as a result of the interaction between the walls [26]. Here, the energy of interaction between the walls is calculated for DWNTs with walls having an optimized structure and with a unit cell of the same average length $t_{a}=\left(t_{2}+t_{1}\right) / 2$.

We determined the energy of interaction between the walls as the difference between the total energy of the DWNT and the total energies of isolated walls. To analyze the characteristics of the relative motion of nanotube walls, we must calculate the dependence of energy $U$ of interaction between two adjacent walls on the coordinates describing the relative position of the walls (angle $\phi$ of the relative rotation of the walls about the nanotube axis and length $z$ of the relative displacement of the walls along this axis).

In accordance with the symmetry of $\left(n_{1}, n_{1}\right) @\left(n_{2}, n_{2}\right)$ DWNTs, the unit cell of the potential relief of the interwall interaction energy of such DWNTs is a rectangle with sides $\delta_{z}=t_{a} / 2$ and $\delta_{\phi}=$ $\pi G C D\left(n_{1}, n_{2}\right) / n_{1} n_{2}$, where $G C D\left(n_{1}, n_{2}\right)$ is the greatest common division of numbers $n_{1}$ and $n_{2}$ [42]. For $(5,5) @(10,10)$ DWNT, we have $\delta_{\phi}=\pi / 10$. The interwall interaction energy $U(z, \phi)$ for $(5,5) @(10,10)$ DWNT was calculated for 100 relative positions of the walls, corresponding to a unit cell of the potential relief. We found that potential energy $U_{\text {int }}$ of the interaction between layers, which corresponds to the minimum of function $U(z, \phi)$, is $14.5 \mathrm{meV}$ per atom of the outer wall. This result is in good agreement with the experimental values of the energy of interaction between graphite layers $(35 \pm 10 \mathrm{meV} / \mathrm{atom})$ [17] and between the walls of multi-walled nanotubes with large diameter (20-33 meV/atom) [2], as well as with the results of calculation by the density functional method in the local density approximation with the basis set of pseudowave $d d d d$ functions $(23.83 \mathrm{meV} /$ atom of the outer wall of a $(5,5) @(10,10)$ DWNT) [26].

The relative positions of the walls corresponding to a higher symmetry of a DWNT are singular points of interwall interaction energy $U(z, \phi)$ (extrema or saddle points) [42]. For such relative positions of the walls, some of the $U_{2}$ axes (binary axes) coincide. The $U_{2}$ axes are perpendicular to the principal DWNT axis and 
pass through the middles of all bonds and through the centers of all hexagons of the structure of the wall. According to our calculations, the $U_{2}$ axes passing through the middles of tilted bonds of the $(5,5)$ wall and the middles of the bonds lying in the plane perpendicular to the DWNT axis of the $(10,10)$ wall layer coincide for the relative position of the walls corresponding to the minimum of the energy of interaction between the walls. The same relative position of the walls corresponding to the minimum of energy $U(z, \phi)$ was obtained using semiempirical calculations [43]. The relative position of the walls corresponding to the energy minimum in our calculations is shifted along the $z$ axis by a half-period $\delta_{z}$ relative to the position obtained from calculations based on the density functional method in the local density approximation with the basis set of pseudowave $d d d d$ functions [26].

Table 1 (see above) contains the calculated values of barriers $\Delta U_{z}$ and $\Delta U_{\phi}$ for relative sliding of the walls along the DWNT axis and relative rotation of the walls (between their relative positions corresponding to the interaction energy minimum), respectively, as well as ratio $\gamma_{b}=\Delta U_{\phi} / \Delta U_{z}$ of these barriers and analogous results from the literature obtained with the help of semiempirical methods and ab initio calculations.

In accordance with calculations based on the density functional method [26] and semiempirical calculations 21], the energy of interaction between commensurate nonchiral DWNT walls $\left(n_{1}, n_{1}\right) @\left(n_{2}, n_{2}\right)$ and $\left(n_{1}, 0\right) @\left(n_{2}, 0\right)$ can be interpolated by the following expression:

$$
U(\phi, z)=U_{0}-\frac{\Delta U_{\phi}}{2} \cos \left(\frac{2 \pi}{\delta_{\phi}} \phi\right)-\frac{\Delta U_{z}}{2} \cos \left(\frac{2 \pi}{\delta_{z}} z\right)
$$

where $U_{0}$ is the mean energy of interwall interaction. Henceforth, we will normalize the values of $U_{0}, \Delta U_{z}$ and $\Delta U_{\phi}$ to an atom of the outer wall. In accordance with our calculations, the shape of barriers for relative sliding and rotation of the walls can also be interpolated by cosinusoids to within the computation error; however, the heights of the barriers for sliding or rotation depend on the relative rotation or shear of walls, respectively, along the DWNT axis. Table 1 contains our results for barrier heights for relative rotations and sliding of the walls at the instant of their passage through the relative position of the walls corresponding to the minimum of the interwall interaction energy. However, the barrier height at the instant of passage through the relative position of the walls corresponding to the energy maximum of interwall interaction is $2 \%$ lower as compared to that for the passage through the minimum, and the barrier height for the sliding is lower than the computational error $(0.001 \mathrm{meV} /$ atom $)$.

The high value of the barrier ratio for relative rotations and sliding of the walls (see Table 1) also indicates a high ratio of threshold forces for relative rotations and sliding of the walls. Consequently, we believe that this nanotube can be used as a slider bearing.

A telescopic extension of the inner wall gives rise of a capillary force $F_{c}$ pulling the inner wall back into the outer wall. The average value of this force is defined as

$$
\left\langle F_{c}\right\rangle=\left\langle\frac{d U}{d l}\right\rangle=\frac{U_{0} 4 n_{2}}{t_{a}},
$$

where $4 n_{2}$ is the number of atoms in the unit cell of the outer wall. The average capillary force for interwall 
interaction energy values $U_{0}$ and length $t_{a}$ of the unit cell of the nanotubes calculated here is $\left\langle F_{c}\right\rangle=0.380$ $\mathrm{nN}$.

Our values of barriers $\Delta U_{z}$ and $\Delta U_{\phi}$ for the relative motion of DWNT walls can be used for calculating the values of a number of physical quantities characterizing the interaction and relative motion of DWNT walls. Expression (5) for the dependence of interwall interaction energy $U(\phi, z)$ on the relative position of the walls defines the threshold static friction forces $F_{z}$ and $F_{\phi}$ for the relative sliding of the walls along the nanotube axis and their relative rotation, respectively:

$$
F_{z}=\frac{4 n_{2} \pi L_{o v} \Delta U_{z}}{\delta_{z} t_{a}}, \quad F_{\phi}=\frac{4 n_{2} \pi L_{o v} \Delta U_{z}}{\delta_{\phi} R_{m} t_{a}},
$$

where $L_{o v}$ is the overlap length of the walls and $R_{m}$ is the radius of the movable wall. The shear strengths for relative sliding of the walls along the nanotube axis and their relative rotation are defined, respectively, as

$$
M_{z}=\frac{F_{z}}{S}, \quad M_{\phi}=\frac{F_{\phi}}{S},
$$

where $S$ is the area of the overlap surface of the walls,

$$
S=2 \pi L_{o v}\left(\frac{R_{1}+R_{2}}{2}\right)=\pi L_{o v}\left(R_{1}+R_{2}\right),
$$

$R_{1}$ and $R_{2}$ are the radii of the inner and outer wall, respectively. In accordance with our calculations, the values of shear strengths for $(5,5) @(10,10)$ DWNT are $M_{z}=1.0 \pm 0.2 \mathrm{MPa}$ and $M_{\phi}=51.5 \pm 0.5 \mathrm{MPa}$, respectively, for the relative sliding of the walls along the nanotube axis and their relative rotation. In calculating the shear strengths for the relative rotation of the walls, we assumed that the inner wall is mobile.

The equality of forces $F_{c}=F_{z}$ acting on the movable wall controls the minimal overlap length $L_{m}$ of the walls for which the static friction force prevents the inner wall from being pulled by the capillary force:

$$
L_{m}=\frac{\delta_{z} U_{0}}{\pi \Delta U_{z}} .
$$

According to our calculations, $L_{m}=110 \pm 20 \mathrm{~nm}$ for $(5,5) @(10,10)$ DWNT.

To find the frequency of small rotational oscillations and relative vibrations of the walls along the DWNT axis, we approximate expression (5) for the dependence of the interwall interaction energy on their relative position by a parabolic potential well in the vicinity of the minimum $U_{m}$ of this dependence,

$$
U\left(z^{\prime}\right)=U_{m}+\frac{k_{z} z^{\prime 2}}{2}, \quad U\left(\phi^{\prime}\right)=U_{m}+\frac{k_{\phi} \phi^{\prime 2}}{2},
$$

where $z^{\prime}$ and $\phi^{\prime}$ are the coordinate of displacement relative to the energy minimum of interwall interaction. For $\left(n_{1}, n_{1}\right) @\left(n_{2}, n_{2}\right)$ DWNTs, we have

$$
k_{z}=\frac{8 n_{2} L_{o v} \Delta U_{z}}{t_{a}}\left(\frac{2 \pi}{t_{a}}\right)^{2}, \quad k_{\phi}=\frac{8 n_{2} L_{o v} \Delta U_{\phi}}{t_{a}}\left(\frac{n_{1} n_{2}}{G C D\left(n_{1}, n_{2}\right)}\right)^{2} .
$$


Frequencies $\nu_{\phi}$ and $\nu_{z}$ of small relative rotational and axial (along the DWNT axis) oscillations of the walls are defined as

$$
\nu_{z}=\frac{1}{2 \pi} \sqrt{\frac{k_{z}}{M}}, \quad \nu_{\phi}=\frac{1}{2 \pi} \sqrt{\frac{k_{\phi}}{J}},
$$

respectively, where $M$ and $J$ are the reduced mass and moment of inertia of the walls.

In the case when only the inner wall of a DWNT is mobile, its moment of inertia is $J_{1}=M_{1} R_{1}^{2}$, where $M_{1}=4 n_{1} m_{0} L_{1} / t_{a}$ is the mass of the inner wall, $L_{1}$ is its length, and $m_{0}$ is the mass of a carbon atom. Analogously, if only the outer wall is mobile, its moment of inertia and mass are defined as $J_{2}=$ $M_{2} R_{2}^{2}$ and $M_{2}=4 n_{2} m_{0} L_{2} / t_{a}$, where $L_{2}$ is the length of the outer wall. In the case when both walls are mobile, the reduced moment of inertia and the mass of the system are given by $J_{12}=J_{1} J_{2} /\left(J_{1}+J_{2}\right)$ and $M_{12}=M_{1} M_{2} /\left(M_{1}+M_{2}\right)$. The latter case can be encountered, for example, in zero-gravity conditions in a space-based laboratory.

The frequencies of rotational and axial relative oscillations of the walls are independent of the DWNT length when the lengths of both walls are identical, or when a shorter wall is mobile and the longer wall is fixed. In these cases, the overlap length of the walls coincides with the length of the mobile wall, and the expressions of the frequencies of relative axial oscillations of the walls assume the form

$$
\begin{gathered}
\nu_{z, 1}=\frac{1}{t_{a}} \sqrt{\frac{2 n_{2} \Delta U_{z}}{n_{1} m_{0}}}, \quad \nu_{z, 2}=\frac{1}{t_{a}} \sqrt{\frac{2 \Delta U_{z}}{m_{0}}}, \\
\nu_{z, 12}=\frac{1}{t_{a}} \sqrt{\frac{2\left(n_{1}+n_{2}\right) \Delta U_{z}}{n_{1} m_{0}}} .
\end{gathered}
$$

Here, $\nu_{z, 1}, \nu_{z, 2}$, and $\nu_{z, 12}$ correspond to the inner, outer, and both mobile walls. The expressions for frequencies $\nu_{\phi, 1}, \nu_{\phi, 2}$, and $\nu_{\phi, 12}$ of relative rotational oscillations of the walls corresponding to the inner, outer, and both mobile walls, respectively, assume the form

$$
\begin{gathered}
\nu_{\phi, 1}=\frac{n_{2}}{\pi G C D\left(n_{1} n_{2}\right) R_{1}} \sqrt{\frac{n_{1} n_{2} \Delta U_{\phi}}{2 m_{0}}}, \quad \nu_{\phi, 2}=\frac{n_{1} n_{2}}{\pi G C D\left(n_{1} n_{2}\right) R_{2}} \sqrt{\frac{\Delta U_{\phi}}{2 m_{0}}} \\
\nu_{\phi, 12}=\frac{n_{2}}{\pi G C D\left(n_{1} n_{2}\right) R_{1} R_{2}} \sqrt{\frac{n_{1}\left(n_{1} R_{1}^{2}+n_{2} R_{2}^{2}\right) \Delta U_{\phi}}{2 m_{0}}} .
\end{gathered}
$$

As a result of our ab initio calculations, we obtained the following values of frequencies of relative oscillations of the walls: $\nu_{z, 1}=1.74 \pm 0.17, \nu_{z, 2}=1.22 \pm 0.12, \nu_{z, 12}=2.1 \pm 0.2 \nu_{\phi, 1}=12.66 \pm 0.06$, $\nu_{\phi, 2}=4.50 \pm 0.02$ and $\nu_{\phi, 12}=13.44 \pm 0.07 \mathrm{~cm}^{-1}$.

If the energy of thermal motion of a short mobile wall of length $L$ is much lower than the height of the barriers for the sliding of this wall along the DWNT axis $\left(k T \ll 4 n_{2} \Delta U_{z} L / t_{a}\right)$ and/or for its rotation $\left(k T \ll 4 n_{2} \Delta U_{\phi} L / t_{a}\right)$, then hopping diffusion of the shorter wall along the DWNT axis and/or its hopping rotational diffusion, respectively, takes place [9, 10]. In this case, diffusion coefficients $D_{z}$ and $D_{\phi}$ for the motion of the mobile wall along the axis and for rotational diffusion are defined as 


$$
\begin{aligned}
& D_{z}=\frac{1}{2} \Omega_{z} \delta_{z}^{2} \exp \left(-\frac{4 n_{2} \Delta U_{z} L}{k T t_{a}}\right) \\
& D_{\phi}=\frac{1}{2} \Omega_{\phi} \delta_{\phi}^{2} \exp \left(-\frac{4 n_{2} \Delta U_{\phi} L}{k T t_{a}}\right)
\end{aligned}
$$

respectively, where $\Omega_{z}$ and $\Omega_{\phi}$ are the preexponential factors in the Arrhenius formula for the frequency of the jump of the mobile wall between equivalent minima of interwall interaction energy.

Simulation based on the molecular dynamics method shows that the preexponential factor in the Arrhenius formula for the frequency of the jump of a $\mathrm{C}_{60} @ \mathrm{C}_{240}$ nanoparticle shell between equivalent energy minima of interwall interaction is $\Omega=540 \pm 180 \mathrm{GHz}$ [44]. The frequency of small relative rotations near the minima of interwall interaction energy calculated here for such a nanoparticle is $\nu=60 \mathrm{GHz}$ for the same shape of the shells and the same potential of interaction between atoms of the shells. Thus, ratio $\Omega / \nu$ of the preexponential factor in the Arrhenius formula to the frequency of small oscillations is approximately 10 . We believe that ratio $\Omega / \nu$ for other carbon nanostructures with embedded graphite layers (in particular, carbon nanotubes) is on the same order of magnitude. We used the value of $\Omega / \nu \sim 10$ for estimating the diffusion coefficients of walls in $(5,5) @(10,10)$ DWNT on the basis of our ab initio calculations. The values for the diffusion coefficients of a short mobile wall of length $L=100 \mathrm{~nm}$ along the DWNT axis at a temperature of $300 \mathrm{~K}$ are $D_{z, 1} \approx 8 \cdot 10^{-12} \mathrm{~m}^{2} / \mathrm{s}$ and $D_{z, 2} \approx 6 \cdot 10^{-12} \mathrm{~m}^{2} / \mathrm{s}$ for the inner and outer wall, respectively.

Under the action of force $F_{d}$ directed along the DWNT axis, the shorter wall may drift along the longer one at a velocity $v=B_{z} F_{d}$, where $B_{z}$ is the mobility of the shorter wall for its motion along the DWNT axis. In the case of a small force $\left(F_{d} \delta_{z} \ll 2 \Delta U\right.$, where $\Delta U$ is the barrier height for the motion of the shorter wall along the DWNT axis), mobility $B_{z}$ is related to the diffusion coefficient by the Einstein relation $D_{z}=k T B_{z}$ 9, 10]. According to our estimates, for the drift of a wall $100 \mathrm{~nm}$ in length at a velocity of $0.1 \mathrm{~m} / \mathrm{s}$ at a temperature of $T=300 \mathrm{~K}$, forces of $F_{d, 1} \approx 50 \mathrm{pN}$ and $F_{d, 2} \approx 70 \mathrm{pN}$ must be applied to the inner and outer walls, respectively.

Rotational diffusion for walls of length $L=100 \mathrm{~nm}$ at a temperature of $300 \mathrm{~K}$ in $(5,5) @(10,10)$ DWNT is ruled out in view of the large height of the barrier for the relative rotation of the walls. For a mobile wall of length $L=10 \mathrm{~nm}$, the values of rotational diffusion coefficients at $300 \mathrm{~K}$ are $D_{\phi, 1} \approx 2 \cdot 10^{4} \mathrm{rad}^{2} / \mathrm{s}$ and $D_{\phi, 2} \approx 6 \cdot 10^{3} \mathrm{rad}^{2} / \mathrm{s}$ for diffusion of the inner and outer wall, respectively.

A theory of the commensurability-incommensurability phase transition in DWNTs with nearly commensurate walls has been developed recently [11]. In accordance with this theory, such a DWNT can be either in a commensurate phase with identical lengths of the unit cells of the walls, or in an incommensurate phase, in which long segments with practically commensurate walls alternate with relatively short incommensurability defects. The phase of the DWNT is controlled by the value of the incommensurability parameter

$$
h=\frac{2 n_{2} \Delta U_{z}}{\mu_{12}(\Delta t)^{2}}, \quad \mu_{12}=\frac{\mu_{1} \mu_{2}}{\mu_{1}+\mu_{2}}
$$


where $\mu_{1}$ and $\mu_{2}$ are the elastic coefficients of the inner and outer wall, respectively, with the length of a DWNT unit cell.

It should be noted that the incommensurability parameter is the ratio of the difference in energy of interwall interaction for the commensurate phase and a completely incommensurate state to the elastic energy of the DWNT in the commensurate phase (both values of energy are normalized to a DWN unit cell). The DWNT is in a commensurate phase if $h>h_{c}=\pi^{2} / 8$ and in an incommensurate phase if $h<h_{c}$. In accordance with our calculations, $h=0.13 \pm 0.03$ for $(5,5) @(10,10)$ DWNT. Thus, this DWNT is in an incommensurate phase. In this phase, the DWNT has the following structure: commensurate regions with the same length of the unit cells of the walls alternate with incommensurability defects. In each incommensurability defect, the number of unit cells in the wall with a shorter equilibrium length of the unit cell is greater by unity than the corresponding number in the other wall (incommensurability defects are analogous to dislocations in a crystal). The expression for the length of an incommensurability defect assumes the form [1]

$$
l_{d}=\frac{t_{a}}{2} \sqrt{\frac{\mu_{12} t_{a}^{2}}{8 n_{2} \Delta U_{z}}}
$$

Our calculations give an estimate of $l_{d}=146 \pm 21 \mathrm{~nm}$.

\section{CONCLUSIONS}

Let us consider how to experimentally verify our results. At present, the upper boundary of the shear strength for the relative sliding of the walls relative to the nanotube axis determined with the help of atomic force microscopy is $M_{z}<0.04$ [2]. In most cases, the barriers for the sliding of adjacent walls (and, hence, the corresponding shear strength) are negligibly small (due to the incompatibility of translational symmetry of the walls for incommensurate walls [19] and to incompatibility of helical symmetries of the walls for commensurate chiral walls $[18,21,25]$ ). In experiment [2], the shear strength was measured only for a single pair of walls of a multi-walled nanotube, which was characterized by a smaller value of this strength as compared to other pairs of adjacent walls. The chirality indices of the walls were not determined. Thus, we believe that the experimental value of the upper boundary of the shear strength for the relative sliding of the walls corresponds to incommensurate or commensurate chiral walls. However, the barrier for the relative sliding of adjacent walls may attain an appreciable value only for commensurate nonchiral walls (in particular, for $(5,5) @(10,10)$ DWNT) 18, 19, 20, 21]. The shear strengths calculated here for $(5,5) @(10,10)$ DWNT exceed by several orders of magnitude the corresponding boundary of this value (0.04 MPa) measured by an atomic force microscope for the relative sliding of adjacent walls with indeterminate chirality indices. Consequently, the shear strengths for this DWNT can easily be determined experimentally. In our opinion, the minimal overlap length of the walls in the case of the telescopic extension of the inner wall, for which the static friction force prevents the inner wall from being pulled by the capillary force, can also be measured using atomic force microscopy. 
The DWNT images obtained by transmission electron microscopy demonstrate that the interwall interaction may result in extension of one wall and compression of the other wall accompanied by the formation of commensurate regions [45]. We believe that transmission electron microscopy can also be used for observing incommensurability defects in DWNTs.

The calculated frequencies of relative oscillations of the walls can be measured using terahertz spectroscopy or Raman spectroscopy. It should be noted that Raman spectra have recently been obtained for isolated nanotubes [46]. In such measurements, the frequencies of relative oscillations of the walls should be distinguished from the frequencies of other DWNT modes corresponding to those in isolated walls. In accordance with our calculations, the modes of $(5,5) @(10,10)$ DWNT with the lowest frequencies, which correspond to the modes existing in isolated walls, have frequencies exceeding $40 \mathrm{~cm}^{-1}$ [47, 48]. Thus, the frequencies of relative oscillations of the walls in $(5,5) @(10,10)$ DWNT correspond to another frequency range as compared to the frequencies of the remaining modes of this DWNT.

Since the barriers for the relative motion of the walls are controlled by the chirality indices of the walls 8, 18, 19, 20, 21, 25, 26], the measurements of the shear strengths for the relative motion of the walls and the frequencies of their relative oscillations should be accompanied by determination of the chirality indices of the walls. It was shown that the chirality indices of both walls of a DWN can be determined using electron diffractometry [49]. We believe that the characteristics of $(5,5) @(10,10)$ DWNT obtained here on the basis of $a b$ initio calculations of the interwall interaction energy can be determined by contemporary experimental techniques. Such measurements would facilitate progress in developing theoretical methods for studying the interwall interaction in nanotubes, as well as in developing NEMSs based on the relative motion of the walls of nanotubes.

\section{ACKNOWLEDGMENTS}

This work has been partially supported by the Russian Foundation of Basic Research (grants 08-02-00685 and 08-02-90049-Bel)

[1] J. Cumings and A. Zettl, Science 289, 602 (2000).

[2] A. Kis, K. Jensen, S. Aloni et al., Phys. Rev. Lett. 97, 025501 (2006).

[3] A.M. Fennimore, T.D. Yuzvinsky, W.Q. Han et al., Nature 424, 408 (2003).

[4] B. Bourlon, D.C. Glatti, L. Forró and A. Bachtold, Nano Lett. 4, 709 (2004).

[5] V.V. Deshpande, H.-Y. Chiu, H.W. Ch. Postma et al., Nano Lett. 6, 1092 (2006).

[6] Q. Zheng and Q. Jiang, Phys. Rev. Lett. 88, 045503 (2002).

[7] Z.C. Tu and X. Hu, Phys. Rev., 72, 033404 (2005).

[8] R. Saito, R. Matsuo, T. Kimura et al., Chem. Phys. Lett. 348, 187 (2001). 
[9] Yu.E. Lozovik, A.V. Minogin and A.M. Popov, Phys. Lett. A 313, 112 (2003).

[10] Yu. E. Lozovik, A. V. Minogin, and A. M. Popov, Pis'ma Zh. Éksp. Teor. Fiz. 77, 759 (2003) [JETP Lett. 77, 631 (2003)].

[11] E. Bichoutskaia, A.M. Popov, M.I. Heggie and Yu.E. Lozovik, Fullerenes, Nanotubes and Carbon Nanostructures 14, 131 (2006).

[12] E. Bichoutskaia, A.M. Popov, Y.E. Lozovik et al., Phys. Lett. A 366, 480 (2007).

[13] A.M. Popov, E. Bichoutskaia, Yu.E. Lozovik and A.S. Kulish, Phys. Stat. Sol. (a) 204, 1911 (2007).

[14] Yu. E. Lozovik and A. M. Popov, Usp. Fiz. Nauk 177, 786 (2007) [Phys.-Usp. 50, 749 (2007)].

[15] R. Saito, M. Fujita, G. Dresselhaus, M.S. Dresselhaus, Appl. Phys. Lett., 60, 18, 2204 (1992).

[16] R.A. Jishi, M.S. Dresselhaus, G. Dresselhaus, Phys. Rev. B, 47, 24, 16671 (1993).

[17] L.X. Benedict, N.G. Chopra, M.L. Cohen et al., Chem. Phys. Lett. 286, 490 (1998).

[18] A.N. Kolmogorov and V.H. Crespi, Phys. Rev. Lett. 85, 4727 (2000).

[19] M. Damnjanović, T. Vuković and I. Milošević, Eur. Phys. J. B 25, 131 (2002).

[20] T. Vuković, M. Damnjanović and I. Milošević, Physica E 16, 256, (2003).

[21] A.V. Belikov, A.G. Nikolaev, Yu.E. Lozovik and A.M. Popov, Chem. Phys. Lett. 385, 72 (2004).

[22] J.-C. Charlier and J.P. Michenaud, Phys. Rev. Lett. 70, 1858 (1993).

[23] Y.K. Kwon and D. Tomanek, Phys. Rev. B 58, 16001(R) (1998).

[24] A.H.R. Palser, Phys. Chem. Chem. Phys. 1, 4459 (1999).

[25] E. Bichoutskaia, A.M. Popov, A. El-Barbary et al., Phys. Rev. B 71, 113403 (2005).

[26] E. Bichoutskaia, A.M. Popov, M.I. Heggie and Yu.E. Lozovik, Phys. Rev. B 73, 045435 (2006).

[27] T.W. Ebbesen and P.M. Ajayan, Nature 358, 220 (1992).

[28] J.L. Hutchison N.A. Kiselev, E.P. Krinichnaya et al., Carbon 39, 761 (2001).

[29] L. Ci, Z. Pao, Z. Zhou, D. Tang et al., Chem. Phys. Lett. 359, 63 (2002).

[30] S. Bandow, M. Takizawa, K. Hirahara et al., Chem. Phys. Lett. 337, 48 (2001).

[31] J. Sloan, R.E. Dunin-Borkowski, J.L. Hutchison et al., Chem. Phys. Lett. 316, 191 (2000).

[32] D.M. Ceperley and B.J. Alder, Phys. Rev. Lett. 45, 566 (1980).

[33] G. Kresse and J. Furthmüller, Phys. Rev. B 54, 11169 (1996).

[34] G. Kresse and J. Hafner, J. Phys.: Condens. Matter 6, 8245 (1994).

[35] H. J. Monkhorst and J. D. Pack, Phys. Rev. B 13, 5188 (1976).

[36] N. Mounet and N. Marzari, Phys. Rev. B 71, 205214 (2005).

[37] G. Van Lier, G. Van Alsenoy, V. Van Doren and P. Geerlings, Chem. Phys. Lett. 326, 181 (2000).

[38] D. Sanchez-Portal, E. Artacho, J.M. Soler et al., Phys. Rev. B 59, 12678 (1999).

[39] S.L. Mielke, D. Troya, S. Zhang et al., Chem. Phys. Lett. 390, 413 (2004).

[40] T. Ozaki, Y. Iwasa and T. Mitani, Phys. Rev. Lett. 84, 1712 (2000).

[41] E. Hernandez, C. Goze, P. Bernier, and A. Rubio, Phys. Rev. Lett. 80, 4502 (1998).

[42] M. Damnjanović, I. Milošević, T. Vuković, and R. Sredanović, Phys. Rev. B 60, 2728 (1999).

[43] M. Damnjanović, E. Dobardzić, I. Milošević et al., New J. Phys. 5, 148.1 (2003). 
[44] Yu.E. Lozovik and A.M. Popov, Chem. Phys. Lett. 328, 355 (2000).

[45] A. Hashimoto, K. Suenaga, K. Urita et al., Phys. Rev. Lett. 94045504 (2005).

[46] L. Zhang, Z. Jia, L. Huang et al., J. Phys. Chem. C 112, 13893 (2008).

[47] E. Dobardžić, I. Milošević, T. Vuković et al., Eur. Phys. J. B 34, 409 (2003).

[48] T. Vuković, S. Dmitrović, and E. Dobardžić, Nanotechnology 17, 747 (2006).

[49] K. Hirahara, M. Kociak, S. Bandow et al., Phys. Rev. B 73, 195420 (2006). 